\title{
Bi Supra Transitive and Semi Minimal Systems
}

\author{
Taha H. Jasim ${ }^{1}$ Mohammed Nokhas Murad $^{2}$ \\ ${ }^{1}$ College of Computer Science and Mathematics, University of Tikrit, Tikrit, Iraq \\ ${ }^{2}$ Mathematics Department, College of Science, University of Sulaymaniyah, Sulaymaniyah, Iraq \\ Email: tahahameed@gmail.com,muradkakaee@yahoo.com
}

How to cite this paper: Jasim, T.H. and Murad, M.N. (2018) Bi Supra Transitive and Semi Minimal Systems. Open Access Library Journal, 5: e4749.

https://doi.org/10.4236/oalib.1104749

Received: July 2, 2018

Accepted: August 10, 2018

Published: August 13, 2018

Copyright $\odot 2018$ by authors and Open Access Library Inc.

This work is licensed under the Creative Commons Attribution International License (CC BY 4.0).

http://creativecommons.org/licenses/by/4.0/

\section{Open Access}

\begin{abstract}
In the present paper, we define and introduce some new class of topological transitive maps called topological semi-transitive, bi-supra transitive maps, semi-minimal systems and study some of its properties. The main results are the following propositions: Every semi-minimal map is a minimal map, but the converse is not necessarily true and every bi-supra transitive map is transitive map.
\end{abstract}

\section{Subject Areas \\ Computer Engineering}

\section{Keywords}

Bi-Supra Topology, Semi Transitive, Semi Minimal Systems, Bi-Supra

Transitive Maps

\section{Introduction}

Let $A$ be a subset of a topological space $(X, \tau)$. The closure and the interior of $A$ are denoted by $C l(A)$ and $\operatorname{Int}(A)$, respectively. A subset $A$ of a topological space $(X, \tau)$ is said to be semi open [1] (Levine 1963), (resp. regular open [2], preopen [3]) if $A \subset C l(\operatorname{Int}(A))$ (resp. $A=\operatorname{Int}(C l(A)), A \subset \operatorname{Int}(C l(A)))$. A set $A \subset X$ is said to be $\delta$-open [4] if it is the union of regular open sets of a space $X$. The complement of semi open is called semi closed and the complement of a regular open (resp. $\delta$-open) set is called regular closed (resp. $\delta$-closed). The intersection of all $\delta$-closed sets of $(X, \tau)$ containing $A$ is called the $\delta$-closure [4] of $A$ and is denoted by $C l_{\delta}(A)$. Recall that a set $S$ is called regular closed if $S=C l(\operatorname{Int}(S))$. A point $x \in X$ is called a $\delta$-cluster point [4] of $S$ if $S \cap U \neq \phi$ for each regular open set $U$ containing $x$. The set of all $\delta$-cluster points of $S$ is called the $\delta$-closure of $S$ and is denoted by $C l_{\delta}(S)$. A 
subset $S$ is called $\delta$-closed if $\delta C l(S)=S$. The complement of a $\delta$-closed set is called $\delta$-open. The family of all $\delta$-open sets of a space $X$ is denoted by $\delta O(X, \tau)$. The $\delta$-interior of $S$ is denoted by $\operatorname{Int}_{\delta}(S)$ and it is defined as follows:

$$
\operatorname{Int}_{\delta}(S)=\{x \in X: x \in U \subseteq \operatorname{Int}(C l(U)) \subseteq S \text { for some open set } U \text { of } X\}
$$

Functions and of course irresolute functions stand among the most important notions in the whole of mathematical science. Various interesting problems arise when one considers openness. Its importance is significant in various areas of mathematics and related sciences. In 1972, Crossley and Hildebrand [5] introduced the notion of irresoluteness. Many different forms of irresolute functions have been introduced over the years. In the present paper, we define and introduce some new class of topological transitive maps called topological semi-transitive, bi supra and study some of its properties.

Spacing, and type styles are built-in; examples of the type styles are provided throughout this document and are identified in italic type, within parentheses, following the example. Some components, such as multi-leveled equations, graphics, and tables are not prescribed, although the various table text styles are provided. The formatter will need to create these components, incorporating the applicable criteria that follow.

\section{Preliminaries and Definitions}

Norman Levin [1] has developed a few properties of semi-open sets and has defined semi-continuity in terms of them but has not developed a topology on them. This report is an attempt to carry his development further.

Definition 2.1. Recall that a set $A$ in $(X, \tau)$ is said to be semi open (written S.O.) if there exists an open set $O$ such that $O \subseteq A \subseteq C l(O)$.

Theorem 2.2. [1] A subset $A$ in a topological space $X$ is called S.O. if $A \subseteq C l(\operatorname{Int}(A))$.

Proof:

Necessity: Let $A$ be S.O.; then $O \subseteq A \subseteq C l(O)$ for some open set $O$. But $O \subseteq \operatorname{Int}(A)$ and thus $C l(O) \subseteq C l(\operatorname{Int}(A))$. Hence $A \subseteq C l(O) \subseteq C l(\operatorname{Int}(A))$. Sufficiency: Let $A \subseteq C l(\operatorname{Int}(A))$. Then for $O=\operatorname{Int}(A), O \subseteq A \subseteq C l(O)$.

Theorem 2.3. Let $\left(A_{\alpha}\right)_{\alpha \in I}$ be a collection of S.O. sets in a topological space $X$. Then $\bigcup_{\alpha \in I} A_{\alpha}$ is S.O. in $X$.

Definition 2.4. [3] Let $X$ be a topological space and $A \subset X . A$ is called pre-open (P-open) in $X$ Iff $A \subseteq \operatorname{Int}(C l(A)) . A$ is called p-closed iff $A^{C}$ is P-open and it is easy to see that $A$ is $\mathrm{P}$-closed set iff $C l(\operatorname{Int}(A)) \subseteq A$.

\section{Definition 2.5.}

Let $X$ be a topological space and $x \in X, A \subset X$. The Point $X$ is called a P-limit point of $A$ if each P-open set containing $X$, contains a point of $A$ distinct from $X$.

We shall call the set of all P-limit points of $A$ the $\mathrm{P}$-derived set of $A$ and denoted it by $A^{\prime p}$. Therefore $x \in A^{\prime p}$ iff for every P-open set $G$ in $X$ such that $x \in G$ implies that $(G \cap A) \backslash\{x\} \neq \phi$. 


\section{Semi-Transitive Functions and Semi-Minimal}

Let $(X, \tau)$ be a topological space. By a semi-system $(S O(X), f)$ we mean a set of all semi-open subsets of $X$ together with irresolute map. $f: X \rightarrow X$. A set $A \subseteq X$ is called $f$-invariant if $f(A) \subseteq A$.

Topologically semi-transitive and existence of a semi-dense orbit are two notions that play an important rule in every definition of semi-chaos.

\section{Definition 3.1.}

1) Let $(X, \tau)$ be a topological space, a semi-system $(S O(X), f)$ and $f: X \rightarrow X$ irresolute function, then $f$ is said to be a topologically semi-transitive function if for every pair of semi-open sets $U$ and $V$ in $X$ there is a positive integer $n$ such that $f^{n}(U) \cap V \neq \phi$.

2) Let $(X, \tau)$ be a topological space, and $f: X \rightarrow X$ a continuous function, then $f$ is said to be a topologically transitive function if for every pair of open sets $U$ and $V$ in $X$ there is a positive integer $n$ such that $f^{n}(U) \cap V \neq \phi$.

3) A semi-system $(S O(X), f)$ is said to be semi-minimal if $X$ does not contain any non-empty, proper, semi-closed $f$-invariant subset. In such a case we also say that the map $f$ itself is semi-minimal.

Given a point $x$ in a semi-system, $(S O(X), f), O_{f}(x)=\left\{x, f(x), f^{2}(x), \cdots\right\}$ denotes its forward orbit (by an orbit we mean a forward orbit, and $\omega_{f}(x)$ denotes its $\omega$-limit set, i.e. the set of semi-limit points of the sequence $x, f(x), f^{2}(x), \cdots$.

Now, we introduce a new definition on minimal functions called semi-minimal and we study some new theorems associated with this new definition.

A semi-system $(S O(X), f)$ is called semi-minimal if $X$ does not contain any non-empty, proper, s-closed $f$-invariant subset. In such a case we also say that the map $f$ itself is semi-minimal. Another definition of semi-minimal function is that if the orbit of every point $x$ in $X$ is semi-dense in $X$ then the map $f$ is said to be semi-minimal. Let us introduce and study an equivalent new definition.

Definition 3.2. (Semi-minimal) Let $X$ be a topological space, $S O(X)$ be the set of all semi-open sets in $X$ and be irresolute function on $X$. Then $(S O(X), f)$ is called semi-minimal system (or $f$ is called semi-minimal function on $X$ ) if one of the three equivalent conditions hold:

1) The orbit of each point of $X$ is semi-dense in $X$.

2) $\mathrm{Cl}_{s}\left(O_{f}(x)\right)=X$ for each $x \in X$.

3) Given $x \in X$ and a nonempty semi-open $U$ in $X$, there exists $n \in N$ such that $f^{n}(x) \in U$.

Theorem 3.3. For $(S O(X), f)$ the following statements are equivalent:

1) $f$ is an semi-minimal function.

2) If $E$ is an semi-closed subset of $X$ with $f(E) \subset E$, we say $E$ is invariant. Then $E=\phi$ or $E=X$.

3) If $U$ is a nonempty semi-open subset of $X$, then $\bigcup_{n=0}^{\infty} f^{-n}(U)=X$.

\section{Proof:}

1) $\Rightarrow 2$ ): If $A \neq \phi$, let $x \in A$. Since $A$ is invariant and semi-closed, i.e. 
$C l_{s}(A)=A$ so $C l_{s}\left(O_{f}(x)\right) \subset A$. On other hand $C l_{s}\left(O_{f}(x)\right)=X$.

Therefore $A=X$.

$2) \Rightarrow 3)$ Let $A=X \backslash \bigcup_{n=0}^{\infty} f^{-n}(U)$. Since $U$ is nonempty, $A \neq X$ and Since $U$ is semi-open and $f$ is irresolute, $A$ is semi-closed. Also $f(A) \subset A$, so $A$ must be $\phi$. $f$ is irresolute, $A$ is semi-closed. Also $f(A) \subset A$, so $A$ must be an empty set.

$3) \Rightarrow 1$ ): Let $x \in X$ and $U$ be a nonempty semi-open subset of $X$. Since $x \in X \backslash \bigcup_{n=0}^{\infty} f^{-n}(U)$. Therefore $x \in f^{-n}(U)$ for some $n>0$. So $f^{n}(x) \in U$.

Proposition 3.4. Let $S O(X)$ be a semi-compact space without isolated point, if there exists a semi-dense orbit, that is there exists $x_{0} \in X$ such that the set $O_{f}\left(x_{0}\right)$ is semi-dense then $f$ is topologically semi-transitive.

\section{Proof:}

Let $x_{0}$ be such that $O_{F}\left(x_{0}\right)$ is semi-dense. Given any pair $U, V$ of semi-open sets, by semi-density there exists $\mathrm{n}$ such that $f^{n}\left(x_{0}\right) \in U$, but $O_{F}\left(x_{0}\right)$ is semi-dense implies that $O_{F}\left(f^{n}\left(x_{0}\right)\right)$ is semi-dense, there exists $m$ such that $f^{m}\left(f^{n}\left(x_{0}\right)\right) \in V$. Therefore $f^{m+n}\left(x_{0}\right) \in f^{m}(U) \cap V$. That is $f^{m}(U) \cap V \neq \phi$. So $f$ is topological semi-transitive.

Definition 3.5. A semi-system $(S O(X), f)$ is called topologically semi-mixing if for any pair $U, V$ of non-empty semi-open sets there exists $N \in \mathrm{N}$ such that for all $n \geq N$ we have $f^{n}(U) \cap V \neq \phi$. Topologically semi-mixing conveys the idea that each semi-open set $U$, after iterations of $f$, for each semi-open set $V$, for all $n$ sufficiently large, $f^{n}(U)$ intersects $V$.

Definition 3.6. 1) A map $h: X \rightarrow Y$ is called sr-homeomorphism if it is bijective and the map $h$ and it's inverse are irresolute.

2) Two semi-systems $f: X \rightarrow X$ and $g: Y \rightarrow Y$ are said to be topologically semi-conjugate if there is a sr-homeomorphism $h: X \rightarrow Y$ such that $h \circ f=g \circ h$. We will call $h$ a topological semi-conjugacy.

Now, we proceed to prove an important proposition:

Proposition 3.7. If $f: X \rightarrow X$ and $g: Y \rightarrow Y$ are topologically sr-conjugate. Then

1) $f$ is topologically semi-transitive if and only if $g$ is topologically semi-transitive;

2) $f$ is semi-minimal if and only if $g$ is semi-minimal;

3) $f$ is topologically semi-mixing if and only if $g$ is topologically semi-mixing.

\section{Proof:}

Suppose $f$ is semi-transitive. Let $A, B$ be semi-open subsets of $Y$ (to show $g^{n}(A) \cap B \neq \phi$ for some $\left.n>0\right) . U=h^{-1}(A)$ and $V=h^{-1}(B)$ are semi-open subsets of $X$ since $h$ is an irresolute.

Then there exists some $n>0$ such that $f^{n}(U) \cap V \neq \phi$ since $f$ is semi-transitive. Thus (as $f \circ h^{-1}=h^{-1} \circ g$ implies $f^{n} \circ h^{-1}=h^{-1} \circ g^{n}$ ).

$$
\phi \neq f^{n}\left(h^{-1}(A)\right) \cap h^{-1}(B)=h^{-1}\left(g^{n}(A)\right) \cap h^{-1}(B)
$$

Therefore, $\quad h^{-1}\left(g^{n}(A) \cap B\right) \neq \phi \quad$ implies $\quad g^{n}(A) \cap B \neq \phi$ since $h^{-1}$ is invertible. 


\section{Proof:}

Assume that $f: X \rightarrow X$ and $g: Y \rightarrow Y$ are topological semi-systems which are topologically sr-conjugated by $h: Y \rightarrow X$. Thus, $h$ is sr-homeomorphism (that is, $h$ is bijective and thus invertible and both $h$ and $h^{-1}$ are irresolute) and $h \circ g=f \circ h$ that is, the following diagram commutes:

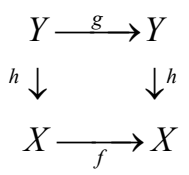

We show that if $g$ is semi-minimal, then $f$ is semi-minimal. We want to show that for any $x \in X, O_{f}(x)$ is semi-dence. Since $h$ is surjective, there exists $x \in X$ such that $y=h^{-1}(x)$. Since $g$ is semi-minimal, $O_{g}(y)$ is semi-dence. For any non-empty semi-open subset $U$ of $X, h^{-1}(U)$ is an semi-open set in $X$ since $h^{-1}$ is irresolute because $h$ is an sr-homeomorphism and it is non-empty since $h$ is invertible. By semi-density of $O_{g}(y)$ there exist $k$ in $N$ such that

$$
g^{k}(y) \in h^{-1}(U) \Leftrightarrow h\left(g^{k}(y)\right) \in U
$$

Since $h$ is s r-conjugacy; as $f \circ h=h \circ g$ implies $f^{k} \circ h=h \circ g^{k} \quad$ so $f^{k}(h(y))=h\left(g^{k}(y)\right) \in U$ thus $O_{f}(h(y))$ intersects $U$. This holds for any non-empty semi-open set $U$ and thus shows that $O_{f}(x)=O_{f}(h(y))$ is semi-dense.

\section{Proof:}

We only prove that if $g$ is topologically semi-mixing then $f$ is also topologically semi-mixing. Let $U, V$ be two semi-open subsets of $X$. We have to show that there is $N>0$ such that for any $n>N, f^{n}(U) \cap V \neq \phi$.

$h^{-1}(U)$ and $h^{-1}(V)$ are two semi-open sets since $h$ is irresolute. If $g$ is topologically semi-mixing then there is $N>0$ such that for any $n>M$, $g^{n}\left(h^{-1}(U)\right) \cap h^{-1}(V) \neq \phi$. Therefore there exits $x \in g^{n}\left(h^{-1}(U)\right) \cap h^{-1}(V)$. That is, $x \in g^{n}\left(h^{-1}(U)\right)$ and $x \in h^{-1}(V)$ if and only if $x=g^{n}(y)$ for $y \in h^{-1}(U)$ and $h(x) \in V$. Thus, since $h \circ g^{n}=f^{n} \circ h$, so that, $h(x)=h\left(g^{n}(y)\right)=f^{n}(h(y)) \in f^{n}(U)$ and we have $h(x) \in V$ that is $f^{n}(U) \cap V \neq \phi$. So, $f$ is semi-mixing.

\section{Bi Supra Transitive and Bi Supra Dense Orbits}

We have studied the relation between definitions of bispra-dense orbit and topological bisupra-transitivity. Now let $X$ be a bisupra space and $f: X \rightarrow X$ bi-irresolute. For more knowledge about transitivity see [6] [7] and [8].

Consider the following two conditions:

(biTT) for every pair of nonempty bisupra-open sets $U$ and $V$ in $X$, there is a positive integer $n$ such that $f^{n}(U) \cap V \neq \phi$.

(biDO) there is a point $x_{0} \in X$ such that $O_{f}\left(x_{0}\right)$ is bisupra-dense.

To study the dynamics of a self-map $f: X \rightarrow X$ means to study the qualitative behavior of the sequences $\left\{f^{n}(x)\right\}$ as $n$ goes to infinity when $X$ varies in $X$, where $f^{n}$ denotes the composition of $f$ with itself $n$ times. 
A subset $E$ of a bisupra space $X$ is said to be of second category in $X$ if $E$ cannot be written as the countable union of subsets which are nowhere bisupra dense in $X$, i.e., if writing $E$ as a union $E=\bigcup_{n \in N} E_{n}$ implies that at least one subset $E_{n} \subset X$ fails to be nowhere bisupra dense in $X$.

Definition 4.1. By a bisupra system we mean a pair $(X, f)$, where $X$ is a bisupra space, and $f: X \rightarrow X$ is a bisupra irresolute function. The dynamics of the system is given by $x_{n+1}=f\left(x_{n}\right), x_{0} \in X, n \in \mathbf{N}$ and the solution passing through $x$ is the sequence $\left\{f\left(x_{n}\right)\right\}$ where $n \in \mathbf{N}$.

Definition 4.2. 1) Let $x \in X$, then the set $\left\{x, f(x), f^{2}(x), \cdots\right\}$ is called an orbit of $x$ under $f$ and is denoted by $O_{f}(x)$, so $O_{f}(x)$ is the set of points which occur on the orbit of $x$ at some positive time, and the sequence $x, f(x), f^{2}(x), \cdots$ is called the trajectory of $x$.

2) Let $X$ be bisupra space, $f: X \rightarrow X,\left\{f^{n}\left(x_{0}\right)\right\}_{n=0}^{\infty}$ be a sequence in $X$, and let $x \in X$. Then $\left\{f^{n}\left(x_{0}\right)\right\}$ converges to $x$ if for all bisupra open sets $U$ containing $x$, there exists $N$ such that for all $n>N$.

Any point with bisupra dense orbit is called a bisupra transitive point. A point which is not bisupra transitive is called bisupra intransitive.

3) (Bisupra Transitivity) Let $X$ be a bisupra space with no isolated point. Given any two bisupra open sets $G$ and $H$ in $X$, there is a point $x \in G$ and an $n>0$ such that $f^{n}(x) \in H$.

4) A system is said to be a bisupra mixing if for every pair $G, H$ of non-empty bisupra open sets, if there exist $N$ such that $f^{n}(G) \cap H \neq \phi$ for all $n>N$. We can show that bisupra mixing implies weak bisupra mixing. But the converse is not nesaccerly true.

Recall that a subset $A$ of a set $X$ is called a bisupra-open set if $A=L \cap F$, where $L$ in $S O(X)$ and $F$ in $P O(X)$. Complements of bisupra-closed sets will be called bisupra-open.

Definition 4.3. A function $f: X \rightarrow X$ is called bisupra-irresolute if the inverse image of each bisupra-open set is a bisupra-open set in $X$.

Let $X$ be a bisupra space. The set of bisupra-irresolute functions on $X$ is denoted by $C^{b i}(X)$.

Definition 4.4. Let $X$ be a topological space. A map $f$ is said to have bisupra-dense orbit if there exists $x \in X$ such that $\operatorname{biCl}\left(O_{f}(x)\right)=X$.

Definition 4.5. Let $X$ be a bisupra space, $f: X \rightarrow X$ be bisupra-irresolute map, then the set $A \subseteq X$ is called bisupra transitive set (rsp. transitive set) if for every pair of non-empty bisupra-open (rsp. open) sets $G$ and $H$ in $X$ with $A \cap G \neq \phi$ and $A \cap H \neq \phi$ there is a positive integer $n$ such that $f^{n}(G) \cap H \neq \phi$.

Proposition 4.6. Every bisupra transitive set is transitive set.

\section{Conclusions}

There are the main results of the paper.

Proposition 5.1. Every semi-transitive map is a transitive map as every, but 
the converse not necessarily true.

Proposition 5.2. Every semi-minimal map is a minimal map, but the converse not necessarily true.

Theorem 5.3. For the system $(S O(X), f)$ the following statements are equivalent:

1) $f$ is an semi-minimal map.

2) If $E$ is a semi-closed subset of $X$ with $f(E) \subset E$, we say $E$ is invariant; then $E=\phi$ or $E=X$.

3) If $U$ is a nonempty semi-open subset of $X$, then $\bigcup_{n=0}^{\infty} f^{-n}(U)=X$.

Theorem 5.4. Every bisupra transitive map is transitive map.

\section{Acknowledgements}

First, thanks to my family for having the patience with me for having taking yet another challenge which decreases the amount of time I can spend with them. Specially, my wife who has taken a big part of that sacrifice, and also Amir, my son who helps me for typing my research. Thanks to all my colleagues for helping me for completing my research.

\section{Conflicts of Interest}

The authors declare no conflicts of interest regarding the publication of this paper.

\section{References}

[1] Levin, N. (1963) Semi-Open Sets and Semi-Continuity in Topological Spaces. American Mathematical Monthly, 70, 36-40. https://doi.org/10.1080/00029890.1963.11990039

[2] Stone, M. (1937) Applications of the Theory of Boolean Rings to General Topology. Transactions of the American Mathematical Society, 41, 374-381. https://doi.org/10.1090/S0002-9947-1937-1501905-7

[3] Mashhour, A.S., Abd El-Monsef, M.E. and El-Deep, S.N. (1982) On Pre-Continuous and Weak Pre-Continuous Mappings. Proceedings of the Mathematical and Physical Society of Egypt, 47-53.

[4] Velicko, N.V. (1968) H-Closed Topological Spaces. American Mathematical Society Translations: Series 2, 78, 102-118. https://doi.org/10.1090/trans2/078/05

[5] Crossley, S.G. and Hildebrand, S.K. (1972) Semi Topological Properties. Fundamenta Mathematicae, 74, 233-254. https://doi.org/10.4064/fm-74-3-233-254

[6] Kaki, M.N.M. (2012) New Types of Transitive Functions and Minimal System. International Journal of Basic and Applied Science, 12, 53-58.

[7] Kaki, M.N.M. (2018) Chaos, Mixing Weakly Mixing and Exactness. OALib Journal, 5, 1-6. http://www.oalib.com/journal

[8] Kaki, M.N.M. (2014) New Conceptions of Transitivity and Minimal Mappings. Science Research, 2, 1-6. 